\title{
Focus on neurovascular interactions
}

\author{
Blood vessels in the nervous system are not simply inert bystanders that only support the metabolic needs of \\ neurons. We present a focus on neurovascular interactions that highlights our emerging knowledge of how these \\ interactions shape neuronal function both in health and disease.
}

T he mammalian brain has an intricate and highly evolved network of vasculature that efficiently meets the high metabolic demand of nervous tissue. The neurovasculature in the CNS consists of a complex set of interactions between endothelial cells, pericytes, fibroblasts, neurons and glia, and is not simply a passive entity that merely provides oxygen and nutrients to the underlying neural tissue, but is instead a dynamic structure that responds to cues and sculpts brain function. For example, blood vessels interact with neural stem and progenitor cells and form a supportive niche around blood vessels that supports neurogenesis. Blood vessels and angiogenic molecules actively participate in the pathogenesis of neurological disorders, tumors and stroke. In this issue, we present a focus on neurovascular interactions, which highlights recent efforts in some of these areas and discusses how advances in understanding these intricate interactions may lead to new treatments.

Brain tumor cells depend on a rich blood supply for their proliferation and interact directly with the neurovasculature. These tumor cells are finely regulated by the neurovasculature and in turn actively modify it. In their review, Anita Hjelmeland, Justin Lathia, Sith Sathornsumetee and Jeremy Rich discuss the interactions between a subset of brain tumor cells called brain tumor stem cells (BTSCs), which show an increased tumor propagation capacity, and components of the neurovasculature. BTSCs are enriched in the environment around the blood vessels (the perivascular niche) and stimulate angiogenesis through the secretion of growth factors. The authors discuss the neurovascular factors that help maintain BTSCs, as well as BTSC regulation of endothelial cells, and highlight ways in which our understanding of brain tumor biology can be translated to patient therapies.

The perivascular niche is not only critical for regulating pathogenic cell proliferation in tumor formation, but is also important for regulating normal adult neurogenesis. On page 1382, Steven Goldman and Zhuoxun Chen review the molecular interactions that are critical to the endothelial regulation of stem and progenitor cells in the brain. They discuss the perivascular signals that support neuronal development and the signaling pathways that promote neurogenesis, neural stem cell expansion, differentiation, and neuronal migration and recruitment. Their review addresses the key observations that have been made in this area, as well as analogous concepts regarding vascular cell contributions to gliogenesis.

Ethan Winkler, Robert Bell and Berislav Zlokovic review the rapidly evolving role of CNS pericytes in health and disease. CNS pericytes have both mesodermal and neuroectodermal origins, and these pericytes are opposed to CNS capillaries and have a critical role in regulating cerebral blood flow. The authors review the developmental origin of pericytes as well as the cross-talk and functional coupling between pericytes and endothelial cells. They discuss advances in our understanding of how pericytes control blood-brain barrier formation and integrity and how they support vascular stability and regulate angiogenesis. Abnormal pericyte function has been seen in several CNS disorders, including diabetic retinopathy and some neurodegenerative disorders, and the authors discuss the implications of recent findings for our understanding of pericytes' involvement in disease.

On page 1390, Erik Storkebaum, Annelies Quaegebeur, Miikka Vikkula and Peter Carmeliet discuss molecular insights into neurological disorders that are caused by either excessive blood vessel growth or improper vessel regression. They focus on two monogenic disorders, cerebral cavernous malformation and hereditary hemorrhagic telangiectasia to illustrate the molecular mechanisms that affect the pathogenesis of these cerebrovascular malformations. They also discuss CADASIL, a syndrome caused by Notch3 mutations, as an example of how reduced vascularization provokes ischemic insults in white matter leading to dementia. Their review also highlights recent advances in understanding how vascular disorders can contribute to neurodegeneration in diseases such as Alzheimer's disease or amyotrophic lateral sclerosis (ALS).

Finally, two perspectives in this focus issue address ischemic injury and advances in stroke research. Much research has focused on glutamatergic mechanisms that lead to ischemic neuronal death. On page 1369, Michael Tymianski discusses alternate molecular processes beyond excitotoxicity that may also critically regulate the deleterious consquences of ischemia. In his perspective, Tymianski focuses on a few examples of these nonglutamatergic mechanisms that contribute to a loss of ionic homeostasis or cellular energy failure following cerebral ischemia, such as those mediated by TRP channels, acid-sensing channels, pannexins and hemichannels. Costantino Iadecola and Josef Anrather review the different ways in which the brain protects itself after ischemic insults on page 1363, and argue that pharmacological interventions or other approaches that mobilize these endogenous neuroprotective programs could invigorate stroke research. They suggest that, unlike therapeutic approaches that are based on counteracting selected pathways of the ischemic cascade, new therapies may need a more holistic approach, and also recruit coordinated neurovascular programs that support cerebral perfusion and promote tissue restoration.

The research described in this focus has made substantial progress toward understanding the interface between neural and vascular systems in neuropathology and, by extension, the importance of these interactions in normal brain function. We hope that these articles will give our readers a sense of the recent research in this area and that they may also inspire further basic and clinical work on these important problems. Finally, we thank Kathleen Dave, for conceiving and commissioning this focus during her time at Nature Neuroscience. 